\title{
La vanité ou le miroir des faux-semblants dans Chaque heure blesse de Raoul Danaho
}

L'Ecclésiaste débute ses réflexions par la formule « Vanité des vanités, vanités des vanités, tout est vanité » et il ajoute : "Quel avantage revient-il à l'homme de toute la peine qu'il se donne sous le soleil ? [...] Ce qui a été, c'est ce qui sera, et ce qui s'est fait, c'est ce qui se fera, il n'y a rien de nouveau sous le soleil. S'il est une chose dont on dise : Vois ceci, c'est nouveau ! cette chose existait déjà dans les siècles qui nous ont précédés » (Ecc, $1: 3$ et 9-11).

Cette affirmation nous convie à considérer les différentes actions de l'humain, notamment les ambitions, les connaissances, les biens matériels, les agitations, les divertissements et, somme toute, c'est la vanité de ces éléments qui est mise en exergue, «toute [cette] peine qu'il se donne sous le soleil ». La vanité, dans sa définition la plus large, nous convie à nous pencher sur la fugacité de la vie, le caractère vain et éphémère de tout ce qui se rapporte aux possessions humaines et terrestres. La littérature, comme toutes les autres formes artistiques, relaye cette représentation et entend souligner la caducité ou la fatuité des actions humaines. Les éléments se gorgent de vie et se vident en parallèle, répondant à un processus de miroitement ou de contrebalancement. Des écrivains tentent d'approcher et de circonscrire la vanité. Saint Augustin, désignant l'humain comme la "chétive partie de [la divine] création, [un] être de boue, promenant sa mortalité, et par elle le témoignage de son péché » (Saint Augustin, 1864, p. 363), associait la vanité de l'existence à la condition pécheresse de l'homme, un état qui entachait par là même chacun de ses actes. Pétrarque ressentait intensément le problème de la destinée humaine, à savoir une incompatibilité entre l'attachement aux choses terrestres et celles qui relèvent du céleste.

Mylène Danglades - docteur en langues et civilisations françaises, enseignante de Lettres à l'Université de Guyane. Adresse pour correspondance : Université de Guyane, Campus de Troubiran, 2091 Route de Baduel, BP 792 - 97337 Cayenne Cedex ; e-mail : mylene.danglades@univ-guyane.fr 
Le Canzoniere, paru en 1923, traduit d'ailleurs le conflit entre l'humain et le divin et l'auteur italien ne se réjouit en rien de la labilité des choses humaines. Dans chacune de ses œuvres, les allusions relatives à la fuite du temps, à notre vie et un monde tout aussi éphémères fusent. Ces éléments engendrent chez lui un profond désarroi, le désir de jouir du temps, des êtres chers et de s'accrocher à ce qui est éternelle. Au début du XVII e siècle, les peintres se focalisent sur "Les Vanités ». Pour traduire la viduité de la vie, certains esquissent des natures mortes basées sur divers objets traduisant l'inutilité des biens, du savoir et du plaisir. Ce seront donc les livres, les instruments scientifiques, les objets d'art, le vin ou les mets qui leur serviront d'assises. Et en ce qui concerne la représentation du pouvoir et de l'argent, ils exploiteront les pièces d'or, les armes, les bijoux, les couronnes et les sceptres. D'autres artistes symbolisent l'écoulement du temps par l'utilisation du sablier, de l'horloge, de la bougie, du clepsydre ou par des bulles de savons, des fleurs, des insectes, des coquillages, tout ce qui rappelle l'évanescence de la vie. Ces éléments, agencés sur la toile, invitent au renoncement et à considérer la fin de la vie. Pour ceux qui oublierait la finalité de ces tableaux, les mots de l'Ecclésiaste cités initialement : "Vanité des vanités, tout est vanité » les leur rappellent de même que la locution latine Memento mori (Souviens-toi que tu vas mourir).

Dans son roman Chaque heure blesse, publié en 1968, Raoul Danaho évoque l'histoire d'un jeune homme, Albert, qui cherche sa propre identité et sa propre place dans le monde. Originaire de Cayenne, il s'est retrouvé à Paris en quête d'évasion, mais cette chimère est vaine. La vie ne présente aucun attrait à ses yeux. Il doit « s'efforcer de vivre » (1968, p. 10). "Effort ou résignation à vivre ?...» (1968, p. 10). L'homme pourrait difficilement statuer et l'auteur de poursuivre : "Albert ne savait plus où il en était. Il comprit seulement, à la coloration mélancolique dont ne se départaient pas ses pensées, que sa fatigue de toujours ne l'avait pas quitté » (1968, p. 10). Albert se sent vide, incapable de fusionner avec le monde environnant et l'écriture de Danaho devient par là même fragmentaire comme pour « réfléchir » la vanité de l'existence.

\section{L'effort prolongé dans la nuit}

Le roman s'ouvre sur une citation de Francis Carco, extraite de L'ombre datant de 1933, qui nous rappelle comment l'individu peut tâtonner, déambuler, en quête d'une autre vie, de signes manifestes, mais que l'entreprise ne semble pas toujours aisée :

Où me portaient mes pas, c’était la même histoire,

J'allais toujours vers les sifflets des trains,

Sur un grand boulevard trouble et peuplé de fantômes.

Là, j’attendais je ne sais qui, je ne sais quoi... (Carco, 1933, p. 359-362) 
L'homme est happé dans un engrenage avec l'utilisation de la tournure « Où me portaient mes pas ». Il est dépossédé de sa volonté et ce sont les évènements, " ses pas ", en l'occurrence, qui s'imposaient à lui, le "portaient ». L'adjectif « même " et l'adverbe " toujours » soulignent la répétitivité des actions. " Les sifflets de train » évoquent les départs, la fuite en avant vers un ailleurs indéfini, des éléments démesurés, troubles et fantasques. La course se solde par une issue pour le moins incertaine : « Là, j'attendais je ne sais qui, je ne sais quoi... ». Le mouvement est interrompu, laisse place à l'attente, à une formule anaphorique négative et à une indétermination manifeste " qui », « quoi » renforcée par les points de suspension. Francis Carco, de son vrai nom Francis Carcopino-Tusoli, d’origine corse, est né à Nouméa en NouvelleCalédonie. Confronté à l'autoritarisme, à la brutalité paternelle et au spectacle récurent des bagnards enchaînés en partance pour l'île de Nou, ses écrits sont empreints d'un certain "goût du malheur ", d'un désenchantement, d'une attirance pour les déshérités et les marginaux de l'existence. Roland Dorgelès a pu dire de lui :

Prisonnier comme eux, [les bagnards de Nouméa], mais prisonnier de lui-même, il n’a jamais pu s’évader. C’est toujours ainsi qu'il a vu le monde, observé les êtres dans une brume de mélancolie que nul rayon de joie ne parvenait à percer. (Bihan, 2012, p. 17)

Cette difficulté à "s'évader » ou à expérimenter la joie nous amène à considérer le personnage principal de Raoul Danaho dans Chaque heure blesse. Albert séveille et est rattrapé par une vérité énoncée dès la première ligne du roman : "Encore un jour de plus qu'il faudra s'efforcer de vivre » $(1968$, p. 9). Ses pensées sont introduites au moyen d'un discours direct avec l'expression à l'imparfait « telle avait été la pensée qui avait accueilli Albert à son réveil ", comme pour nous enrôler dans la fiction. La plainte formulée à haute voix nous renvoie à l'éventualité d'un monologue dramatique, à une temporalité s'inscrivant dans une circularité et qui se joue de l'humain, le diffracte entre deux extrémités, l'avenir et le passé, des moments qui n'existent plus ou pas et qui le détournent illusoirement du présent, ce présent qui le concerne davantage. Cette vaine fuite en arrière ou en avant relève du fait que, selon Pascal :

Nous ne nous tenons jamais au temps présent. Nous anticipons l'avenir comme trop lent à venir, comme pour hâter son cours ; ou nous rappelons le passé pour l'arrêter comme trop prompt : si imprudents, que nous errons dans les temps qui ne sont point nôtres, et ne pensons point au seul qui nous appartient ; et si vains, que nous songeons à ceux qui ne sont rien, et échappons sans réflexion le seul qui subsiste. C'est que le présent, d'ordinaire, nous blesse. Nous le cachons à notre vue, parce qu'il nous afflige ; et, s'il nous est agréable, nous regrettons de le voir échapper. Nous tâchons de le soutenir par l'avenir, et pensons à disposer les choses qui ne sont pas en notre puissance pour un temps où nous n'avons aucune assurance d'arriver. (1964, p. 172) 
La conscience du présent génère des souffrances. L'homme s'en détourne ou redoute de la voir s'estomper et se focalise alors sur le futur hypothétique, « un temps où n'avons aucune assurance d'arriver ". L'homme "imprudent ", en agissant de la sorte, ignore sa condition mortelle, se réfugie dans la dissimulation en cherchant à « arrêter ", à « cacher » à sa vue le présent et il génère ainsi son malheur. Ce regard porté constamment vers l'avenir a permis à Montaigne de formuler ces propos : " Nous ne sommes jamais chez nous, nous sommes toujours au-delà. La crainte, le désir, l'espérance, nous élancent vers l'avenir et nous dérobent le sentiment et la considération de ce qui est, pour nous amuser à ce qui sera, voire quand nous ne serons plus» $(1965, \mathrm{I}, 3)$. Le présent est à prendre en compte pour ce qu'il est et il enrôle l'humain dans un système cyclique. Les jours se succèdent tout comme la nostalgie et la fatigue d'Albert :

" Encore un jour de plus qu'il faudra s'efforcer de vivre ». Telle avait été la pensée qui avait accueilli Albert à son réveil, comme surgie des profondeurs de son être durant le sommeil, elle s’était glissée dans quelque recoin de son cerveau pour guetter l'instant où il ouvrirait les yeux. Elle lui parut, non pas désolante, mais plutôt rassurante. Car il lui trouvait l'attrait d'un beau visage attristé qu'un malade resté longtemps en syncope découvre sur lui en sortant de sa léthargie. Traduisait-elle une vérité essentielle? Effort ou résignation à vivre ?... Albert ne savait plus où il en était. Il comprit seulement, à la coloration mélancolique dont ne se départaient pas ses pensées, que sa fatigue de toujours ne l'avait pas quitté. Sa fatigue née de l'ennui que ne cessait de secréter sa vie, et qui semblait par ce matin de mai plus lourde que jamais. (Danaho, 1968, p. 9)

Le sommeil, assimilé à un état léthargique, s'estompe et l'homme est ramené à sa condition d'existant, de souffrant, de "malade resté longtemps en syncope » et qui sort finalement « de sa léthargie ». Dans cette longue analyse, le narrateur nous brosse le trouble de son personnage, la complexité des sentiments, ce désordre intérieur qui l'emprisonne. C'est précisément la pensée qui l'accueille et non l'inverse. Le sujet subit l'action et les verbes s'enchaînent pour signifier l'emprise de la pensée sur l'individu. Elle est semblable à une entité qui « surgi[t] des profondeurs de son être », qui "s'était glissée dans quelque recoin de son cerveau » dans le but de "guetter l'instant où il ouvrirait les yeux " (Danaho, 1968, p. 9). La pensée se présente naturellement à lui. Elle l'accueille, surgit, se glisse en lui comme s'il s'agissait de lui rappeler sempiternellement son existence au monde. Nietzsche posait antérieurement le postulat de l'éternel retour, en proposant à son lecteur une expérience singulière :

Cette vie, telle que tu la vis et l'a vécue, il te faudra la vivre encore une fois et encore d'innombrables fois; et elle ne comportera rien de nouveau, au contraire, chaque douleur et chaque plaisir et chaque pensée et soupir et tout ce qu'il y a dans ta vie d'indiciblement petit et grand doit pour toi revenir, et tout suivant la même succession et le même enchâ̂nement - et également cette araignée et ce clair de lune entre les arbres, et également cet 
instant et moi-même. L'éternel sablier de l'existence est sans cesse renversé, et toi avec lui, poussière des poussières ! (1997, p. 251-252)

L'individu ne doit pas chercher à changer radicalement le cours de sa vie, mais à revivre " chaque douleur et chaque plaisir et chaque pensée et soupir » sans "rien de nouveau ", selon " la même succession et le même enchaînement ». La répétition de la vie se fait donc à l'identique, sans aucune variation pour Albert, " un jour de plus » accompagnée de cette "fatigue de toujours », qui " ne cessait de secréter sa vie, et qui semblait par ce premier matin de mai plus lourde que jamais... » (Danaho, 1968, p. 9). Le temps pèse fatalement sur l'homme et envahit la sphère de sa chambre à l'image du " rayon de soleil » qui s'y « insinuait lentement ». Mais ce temps, qui se répète à l'infini et enserre Albert dans un étau, esquisse néanmoins quelques notes d'espoir avec une pensée assimilée à "l'attrait d'un beau visage " ou " un rayon de soleil " qui se profile dans une " chambre aux murs froids dont aucune gravure ne rompait la monotonie " (Danaho, 1968, p. 9). L'homme parvient difficilement à distinguer les éléments qui pourraient se confondre et s'interpénétrer. Il se désolidarise de tout cela :

Cette délicate échappée de lumière, émissaire discret de la saison nouvelle et de la joie de vivre, ne rencontrait aucun écho auprès d'Albert. Indifférent au monde extérieur, il demeurait prostré dans son lit, un défilé d'images battant fiévreusement son cerveau. (Danaho, 1968, p. 10)

Le périmètre délimitant la mobilité d'Albert s'amenuise progressivement. Après " la chambre aux murs froids ", le voilà " prostré dans son lit ». L'adjectif réducteur nous renvoie à la misère de l'individu, défini jusqu'à maintenant par un simple prénom, Albert, et à son incapacité de considérer son environnement social et climatique. «La saison nouvelle » ne génère aucun bouleversement au niveau de son corps et de son esprit. Dans ce roman, le cycle des saisons qui s’annonce et sénonce par la « délicate échappée de lumière, émissaire discret », ne joue guère ce rôle de catalyseur permettant l'éclosion d'une personnalité nouvelle chez l'homme, comme nous sommes en droit de l'attendre. "Ce premier matin de mai » est associé à un effroyable débordement de vie, à « des rires épais " (Danaho, 1968, p. 9). Ce passage pourrait être rapproché du constat formulé par Rimbaud dans Une saison en enfer, lorsqu'il indique que «le printemps [lui] a apporté l'affreux rire de l'idiot » (1873, p. 1-2). Les ricanements résonnent et trouvent un écho favorable en pleine nuit dans le cœur d'Albert :

Dès six heures, il a été réveillé par les bavardages intempestifs des commères de la rue. Déjà cette nuit, à peine endormi, il avait été tiré de son sommeil par un grand vacarme. Des cris, des aboiements furieux, des rires épais, des jurons grossiers, des bruits de bouteilles se fracassant sur le pavé. Ce charivari était familier de ses nuits. [...] Cependant Albert trouvait un charme particulier à ces réveils en pleine nuit [...]. (Danaho, 1968, p. 10) 
Si le jour et les premiers rayons solaires n'influent point sur le personnage principal, la nuit le désarticule en le désarrimant de son lieu et suscite d’autres images, d'autres mondes. Elle le plonge en lui-même, et l'invite à réfléchir. «Songer, c'est penser çà et là » (2002, p. 174), atteste Victor Hugo, comme si la nuit permettait d'accéder à l'infini, de percevoir l'origine du visible ou comme le peintre de fermer son œil physique pour ouvrir celui de l'esprit. L'informe monte progressivement à la lumière et c'est ainsi qu'Albert

trouvait un charme particulier à ces réveils en pleine nuit : le silence revenu, il prenait les yeux fermés, une conscience plus aiguë de sa propre nuit, de la nuit immense qu'il portait depuis si longtemps en lui, une nuit qui n'avait pas d'issue, ne débouchait sur aucune aube, aucune aurore. Parfois, il atteignait à des moments de lucidité encore plus bouleversante qu'il savourait avec une âpreté qui tenait d'un sentiment confus de résignation et de désespoir, car il réalisait au plus profond de soi, que sa nuit qu'il croyait si totale, si singulière, n'était qu'une petite tache à peine sombre, en fait, une misérable éclaircie au milieu d'une nuit inconvenablement dense, la grande nuit éternelle qui bientôt l'absorberait dans la solitude d'un fosse, la solitude perdue de la mort. (Danaho, 1968, p. 12)

La lumière et l'obscurité résument en soi la vie. Cette "relation entre jour et nuit n'est pas seulement d'opposition, donc d'exclusion réciproque, mais aussi d'inclusion » (Genette, 1969, p. 103). Dans la peinture comme dans la littérature, la nuit s'appuie sur des contrastes de lumières, de clair-obscur. Elle devient un espace à la fois matériel et mental, propice aux échappées, aux multiples réflexions. La nuit souligne ici le lien entre le monde spirituel des morts et le monde naturel des vivants. L'homme se révèle à lui-même et, selon la définition de Friedrich Nietzsche, il « remarque à temps que ce n'est pas ce qu'il est, mais ce pour quoi il passe qui le soutient ou l'abat : voilà l'origine de la vanité » (1997, p. 897).

Albert cherche à "poursuivre sa route ", " ce tâtonnement dans le noir, cette marche aveugle dans la nuit ", en se réfugiant dans la "rêverie " (Danaho, 1968, p. 13), le "dialogue imaginaire» (Danaho, 1968, p. 14). Il s'imagine, non pas dans « la petite rue de l'Hirondelle, à l'allure médiévale » $(1968$, p. 12) qu'il habitait, mais " déambulant le long du boulevard Saint-Michel » ou se retrouve projeté dans son passé à Cayenne (1968, p. 13). Il opère mentalement un va-et-vient entre le passé et l'avenir, entre la réalité et la fiction, et quelque peu lassé de " poursuivre sa tentative de dialogue avec sa passante idéale », il se livre finalement : « - Ah ! comme c'est absurde ! fit-il, interrompant brusquement son effort de représentation mentale. Je suis de ceux qui passent leur temps à rêver leur vie. On n'a pourtant qu'une vie à vivre et il faudra bien que je me résigne à vivre la mienne » $(1968$, p. 16). Raoul Danaho, après avoir introduit le sujet, nous propose des pistes de réflexions :

Mais était-ce sa faute s'il n'arrivait pas à vivre simplement ? Pourquoi ces arrière-pensées, ces restrictions qu'il avait devant la vie, le spectacle de sa propre vie ? Était-ce l’idée 
de la mort qui le hantait ? Sans doute, ne pouvait-il échapper à l'obsession de sa mort mais cette attitude d'expectative qu'il observait devant la vie provenait essentiellement de ce qu'il considérait comme sans objet le fait même d'exister. Il était possible de s'enrichir, de parvenir au faîte de la puissance, d'être un héros, un sage ou un saint. Mais qu'importait ! Une même pensée, une même question revenait toujours, impérieuse, lancinante : «Et puis après ? après? après ? Serait-on Dieu lui-même quel ennui n'éprouverait-on pas à s'abîmer dans la contemplation perpétuelle de soi et du monde ?» (1968, p. 16-17)

Albert se tient en marge de la vie, la considère comme un spectateur, et l'auteur s'interroge sur les raisons d'une telle pratique. La hantise de la mort est mise en exergue, ainsi que la viduité de la vie. La Rochefoucauld énonçait avec une certaine acuité les propos suivants, renvoyant l'homme à lui-même, à ses semblables et au prisme réfléchissant : «Ce qui nous rend la vanité des autres insupportable, c'est qu'elle blesse la nôtre » (1664, pensée 389). Albert ne parvient pas à se mêler à la vie et se contente de la regarder de sa fenêtre « en spectateur, en esthète, du haut d'un observatoire... pour en avoir une vue panoramique» (Danaho, 1968, p. 17). Il n'y entrevoit pas « une simple curiosité de l'intellect qu'il satisfaisait de la sorte. C'était autrement profond ». Il s'abîmait « dans la contemplation intense d'une rue animée » (1968, p. 32). Il y a lieu de s'interroger une fois de plus sur les raisons de cette curiosité, au même titre que le narrateur :

Au fond pourquoi cherchait-il toujours à savoir ? Une curiosité intense l'avait poussé à tout dévorer pêle-mêle dès l'enfance. Mais sa soif n'avait fait que s'aggraver d'année en année jusqu'à créer en lui un vide immense, intolérable. Chaque parcelle de savoir qu'il enregistrait ne faisait que lui témoigner un peu plus de l'inanité de ses efforts, de l'impossibilité d'appréhender ce que, faute de mieux, il nommait l'Absolu. On ne monte pas impunément à l'assaut de l'infini. Et la masse des connaissances qu'accumulaient les hommes depuis des millénaires ne pouvait rien contre un simple fait d'expérience : tout notre savoir venait se briser contre cette réalité terrible, la certitude atroce que nous avions de notre mort prochaine. (1968, p. 20)

Le désir humain de vivre, de comprendre la vie, d'emmagasiner des connaissances le ramène à un constat, celui de la fragmentation, de la mort. Dès lors, nous sommes en droit de nous demander si la lumière parviendra à atteindre et à irradier le cœur d'Albert ou si la rhétorique de la vanité se distendra pour lui permettre de trouver une voie salutaire.

\section{Une lumière et un chemin accessibles ?}

La vie d'Albert est jonchée d'instants, de pensées fugaces, de quêtes et d'échecs. Ce jeune homme de vingt-trois ans est imprégné de ses lectures. "Un peu comme Julien Sorel que plongeait dans une sorte de ravissement la contemplation des effets 
de Mme de Raynald, Albert éprouvait il ne sait quelle joie trouble à fureter dans la garderobe d'Annie, à respirer ses parfums, à palper le tissu de ses vêtements » (Danaho, 1968, p. 28). Il évoquera d'autres réminiscences livresques, notamment au contact d'une de ses jeunes voisines, qu'il qualifie de "petite fille» $(1968$, p. 45) : « En ce moment, vous me caressez le bras. Par la pensée pourtant je vous repousse au fond de moi-même je me refuse exactement comme je ne sais plus quel personnage de Sartre» (1968, p. 46-47). Les écrivains nourrissent manifestement sa réflexion ou son discours, comme s'il s'agissait de lui donner une consistance, une densité en tant que personnage, lui-même. Ce personnage nous rappellera, comme l'a indiqué Laura Helms dans Le personnage de roman, qu'il est " un être de papier, une créature de mots dont la nature purement verbale ne doit pas être oubliée » (Helms, 2018, p. 8). Pour reprendre la formule de Paul Valery (Tel quel, 1996), Albert, ce " vivant sans entrailles », citera également Les Fleurs du mal de Baudelaire : «J'ai longtemps habité sous de vastes portiques » (Danaho, 1968, p. 48) et lorsque Jocelyne lui en demandera la raison, il répondra : "Pour vous faire comprendre que je suis très vieux ». S’ensuivra alors cet échange :

- Vous ne pensez qu'à travers la littérature?

- Tout est littérature.

- Encore une pensée livresque!

Ils échangèrent longtemps de ces propos, de ces menus riens qui n'offraient d'intérêt que pour eux-mêmes. (Danaho, 1968, p. 49)

La littérature peuple la vie du personnage, orchestre ses conversations ou ses agissements. Elle lui donne le sentiments d'exister, de se rattacher aux mots, comme à une ancre sûre. Les mésaventures sentimentales qu'il relate, relèvent également du récit, de la littérature. L'auteur évoque une "chronique ", un "travail d'archiviste ", " une source intarissable », une " exhumation » d'instants et une " résurrection » :

Au fur et à mesure qu'Albert passait ainsi en revue les menus épisodes de sa vie avec Annie, il sentait s'infiltrer en lui une sourde mélancolie. Faire en pensée la chronique d'un amour, revivre, recréer en soi, en remontant le temps ce qui avait fait la joie, l'indifférence ou la douleur de deux êtres un moment rapprochés, constitue souvent pour l'amant qui s'attache à ce lent travail d'archiviste du cour, une source intarissable de nostalgie. Car cette exhumation des instants que nous avons vécus avec la femme aimée, au début de notre liaison avec elle, ne sera jamais qu'une résurrection manquée. Ces instants morts que nous nous efforçons d'arracher malgré tout au néant, décantés par le souvenir de tout ce qui faisait en réalité leur vie profonde, viennent en faussant notre perspective du passé, nous empêcher de jouir pleinement du présent. Ainsi, nous nous retrouvons dépouillés de notre passé véritable alors que l’avenir déjà nous échappe. (1968, p. 29)

Les éléments sont énoncés, s'ordonnent et s'agencent pour retranscrire de « menus épisodes » de la vie d'Albert avec Annie, une de ses petites amies. Il relatera 
quelques souvenirs dans son journal, débutant à la date du 10 juillet 1954 et s'achevant au 2 mai 1957. Il mentionne à ce sujet ses interrogations quant à son obstination à noter inlassablement les évènements marquants de sa vie :

... 15 août 1954. - Pourquoi m’obstinais-je si longtemps à transcrire les menus évènements de ma vie ? Autrefois, jétais persuadé que faute de tenir un journal le sens de la perfection finirait par sémousser en moi. Faut-il vraiment cet effort graphique de tous les jours pour nous aider à cultiver en nous le désir du bien qui, autrement, s'enliserait dans les sables mouvants de nos tendances terre à terre ? Un journal enregistre impitoyablement nos marches et contre-marches sur la voie de l'idéal. (Danaho, 1968, p. 131)

Tous les tâtonnements de l'homme sont notés et c'est un travail qui s'inscrit sur la durée "longtemps ", " tous les jours ", dans le but manifeste de l'aider au niveau de l'apprentissage du bien. Albert, en infatigable scribe, poursuit une succession dépisodes significatifs de sa vie : « . . 2 mai 1957, à cinq heures de l'après-midi. - Je m'étais juré de ne plus revenir à ce journal. Mais j’y reviens encore une dernière fois » (Danaho, 1968, p. 159). La littérature est mise en exergue et elle aussi est considérée comme une vanité, car elle relève de l'illusion, et en tant qu'art attaché au savoir, à la gloire, aux richesses et à la beauté, il lui appartient de décrire le monde des vanités. Le discours se focalise sur la mort, la déchéance, la fragilité et l'éphémère. Le récit d'Albert associant l'auteur, le lecteur et une instance énonciative cherche à attirer ou à pousser le lecteur dans ses retranchements. Les questions fusent dès les premières pages et tout au long du roman : "Traduisait-elle une vérité essentielle ? Effort ou résignation à vivre ?..." (Danaho, 1968, p. 9) ; "Mais nétait-ce pas après tout vivre que de s'imaginer vivre telle ou telle scène de sa vie ? Et le rêve ne permettait-il pas de vivre sa vie telle qu'on désirait la vivre? (Danaho, 1968, p. 18) ; «Quelles raisons inclinaient doncl'homme à espérer sa survie ? Était-ce sa répugnance à concevoir l'anéantissement irrémédiable de l'intelligence ? Ou l'aspiration qu'il avait à se retrouver dans une autre vie où, délivré du besoin, affranchi du dégoût de soi, il serait comme restitué à lui-même ? Ou encore le désir plus courant de croire en une vie nouvelle, au-delà de la mort, qui le compenserait d'une existence misérable sur la terre? " (Danaho, 1968, p. 77-78). Des alternatives sont suggérées avec la conjonction de coordination « ou »; l'adverbe «peut-être » marque la possibilité, le doute, léventualité soulignant ainsi l'instabilité de l'univers social. Nous sommes confrontés à un jeu entre l'apparence et la réalité. Le personnage d'Albert fait surgir « son propre chaos, ses incohérences intérieures » (Danaho, 1968, p. 32). Il évoque la nuit qui semble le traquer : " Mais il songea brusquement à la nuit, cette longue nuit qui allait sétendre devant lui, et au cours de laquelle il était à peu près sûr de ne pas fermer l'oil » (Danaho, 1968, p. 37), et un malencontreux constat, à savoir « ce triste privilège qu'il avait de tout assombrir autour de lui ! » (Danaho, 1968, p. 37). Le personnage affirme sa présence et, en parallèle, sa dissolution. Il est présenté tantôt comme un être labile ou taciturne, tantôt comme un misérable pêcheur, accusé de divers travers ou un « peseur d'âmes » (Danaho, 1968, p. 37). 
On lui avait à maintes reprises, reproché son étrange vocation de peseur d'âmes. Pourquoi ne goûtait-il pas simplement les choses de la vie ? De quel droit se chargeait-il de sonder les reins et les cœurs? [...]

- Vois-tu, lui affirmait péremptoirement un jour un condisciple de la Sorbonne, les êtres comme toi ne sont d'aucune utilité à la société. Ils sont les ennemis jurés de la joie. Tu prends je ne sais quel malin plaisir à tout figer, tout glacer autour de toi. Ne penses-tu qu'il vaille mieux chercher à dérider les autres, les faire s'oublier un instant, plutôt que de te complaire dans ce métier d'analyste, de dissecteur impénitent ? (Danaho, 1968, p. 37-38)

L'image du prophète et du guide, de l'autorité morale accusatrice se profile, se module en fonction des points de vue et des scènes envisagées. La jeune femme, Jocelyne, qu'Albert surprendra dans son sommeil, alors que sa porte était entre-baillée déplore sa folie :

- Avouez que je suis bien bonne de rester là à écouter vos sornettes. Il n'y a pas le moindre grain de bon sens dans tout ce que vous dites. J'aurais dû alerter le patron de l'hôtel et vous faire jeter hors de chez moi avec perte et fracas. (Danaho, 1968, p. 43)

Après ce premier échange, « ils bavardèrent longtemps » et évoquèrent précisément l'art, la musique, la littérature, la philosophie :

Ils brûlaient de la même ardeur des discussions philosophiques. Ils partageaient le même goût, la même soif d'aller jusqu'au fond des choses. Albert trouvait même que sur ce point elle exagérait. Elle voulait tout expliquer, tout clarifier. Il demeurait, lui, persuadé qu'il fallait laisser parfois flotter autour des choses comme des franges d'interférence, des zones indécises, où l'ombre et la lumière, le mystère et l'explicable s'interpénétraient à loisir. (Danaho, 1968, p. 43)

En se référant aux arguments d'Albert, les éléments ne sont pas figés en soi ou résolument antithétiques. "L'ombre et la lumière, le mystère et l'explicable s'interpénètr[ent] à loisir », la vie génère des rencontres comme toutes celles auxquelles se réfèrent le jeune homme, et

il importait de faire en sorte que l'on prit à la vie tout ce qu'elle pouvait raisonnablement nous offrir. L'essentiel était de bien meubler l'intervalle séparant la naissance de la mort et surtout d'aider, dans la mesure de ses moyens, les autres à le franchir. [...] Chacun pouvait, sans avoir la puissance créatrice de ces génies [les grands musiciens], rendre moins douloureuse la progression de son semblable vers la nuit éternelle. (Danaho, 1968, p. 81)

La naissance et la mort constituent deux étapes dans la vie humaine, et la dynamique textuelle s'inscrit précisément dans ce cadre. Albert tâtonne entre la vie 
et la mort et de la même manière qu'il pouvait "s'efforcer de vivre ", ce dernier pouvait jouir du soleil « qui prenait largement possession dans sa chambre».

Albert rejeta ses couvertures et se leva. Ayant terminé sa toilette, il s'approcha de la fenêtre ouverte. Alors la lumière l'enveloppa tout d'un coup et il éprouva la sensation heureuse de la chaleur sur son torse nu qu'avait rafraîchi l'eau froide du robinet. Il eut l'impression que des effluves, un flux magnétique le traversaient. Il respira à fond. Un flot de joie surgi, il ne savait de quelle partie de son être, l'inonda brusquement :

- Ce sont peut-être de tels instants qui donnent du prix à la vie, pensa-t-il. (1968, p. 30)

Cette vie que le jeune homme sévertuait à fuir présente des attraits lorsqu'il y réfléchit à maintes reprises et qu'il se plonge dans l'étude de Corps et Ames de Maxence Van der Meersch, et relève ce passage :

Souffrir pour les autres, leur montrer le chemin du vrai, expier pour eux, c'est dans l'ordre! Être tout seul à soulever les insultes ou les rires, passer pour un fou, c'est dansl'ordre! Tout cela est bon, beau, bien fait ! Cela c'est vivre. (Danaho, 1968, p. 136)

Les trois verbes essentiels, employés par l'auteur pour définir la vie, sont « souffrir ", " montrer » et " expier », des verbes associés à l'humain et l'existence, elle, équivaut à un chemin. Le bonheur suscite autant de réflexion de sa part et des officiers au mess, quand il est enrôlé dans l'armée. Il formule, en ces termes, sa théorie : «Le bonheur [...], c'est un état où l'homme n'a plus à se soucier de l'espace et du temps qui sont abolis, anéantis » (1968, p. 147). Et il ajoute pour rendre plus clair sa pensée : "Les deux tourments majeurs de l'être humain sont la hantise du temps et la nostalgie de l'ailleurs. Supprimez l'espace et le temps et il sera heureux» (1968, p. 147).

Ces réflexions émanant d'Albert montrent l'évolution du personnage, philosophe. Il reprend le discours d'écrivains comme Pascal et Montaigne sur le passé, le présent et l'avenir. L'homme tenterait une fuite en avant au lieu de s'en tenir au présent et à lui-même. La vanité rattachée au temps, à des éléments évanescents apparait donc comme une pensée qui se nourrit d'elle-même ou qui donne parfois l'impression de s'auto-engendrer. L'utilisation accumulative et hyperbolique de lieux communs pose le postulat du bonheur comme un argument d'autorité, une vérité indubitable.

Il est un moment dans la vie [...] où il faut venir aux explications ultimes, où il y a lieu de se mettre à nu en face de soi-même. Il n'est dès lors possible ni de bluffer ni de mentir. Ce moment est arrivé pour toi. Jusqu'ici ta vie, malgré les apparences n’a pas été particulièrement réussie. Pourquoi ? Pourquoi n'es-tu pas heureux?

[...] - La vérité est que j'ai toujours placé le bonheur si haut que je n'aurais pas pu l'avoir par aucune action humaine.

- C’est un tort que d'avoir cru qu'il était hors de toi-même. 
[...] - Être heureux, c'est dominer les apparences. C'est voir les choses telles qu'on veut qu'elles soient dans leur réalité intime. C'est se hisser au niveau de Dieu. En un mot, le bonheur est une véritable et perpétuelle récréation du monde. (1968, p. 180-181)

Le bonheur, au-delà de tous les mots ou formules, ne se conçoit pas sans « une certaine discipline intérieure » et selon les données émises, « [i]l ne réside qu’en nous. Pour le découvrir, il appartient à chaque individu de fournir un effort plus ou moins grand selon son tempérament. L'insatisfaction foncière que certains portent en eux n'est qu'une forme aiguë de cette tendance fâcheuse qu'ont la plupart des hommes à rechercher le bonheur en dehors d'eux » (1968, p. 183). Sur le point d'être happé par l'éternité, Albert « entendait, comme exécutées par un orchestre intérieur, les mesures chargées d'un bonheur ineffable de la 33e Symphonie de Mozart. Jamais, [ajoute l'auteur] une joie aussi profonde ne l'avait traversé. Son cœur pouvait cesser de battre. Il ferma les yeux... » (Danaho, 1968, p. 188-189).

\section{Conclusion}

La vanité peut difficilement se concevoir comme un élément figé dans le temps. Elle englobe de multiples domaines et toute étude approfondie équivaudrait quasiment à une déconstruction en série de l'assemblage friable. La vanité s'auréole d'un voile mystique. Cette mystification et ce mystère invitent à un déchiffrement, à une élucidation, qui sont l'objet d'une véritable fascination. Le propre du mystère dans Chaque heure blesse de Raoul Danaho est de cristalliser l'attention sur le personnage d'Albert, son mode de vie et ses idéologies. Les frasques de l'existence maintiennent le personnage dans une certaine forme de nuit prolongée. Il se débat et tente de trouver des réponses aux questionnements et aux turpitudes de l'existence. Des raisonnements peuvent paraitre vains, illusoires pour expliquer les contingences de la vie. L'utilisation des mots, des effets, des images pour évoquer la vanité relèvent somme toute de la rhétorique de la séduction et de la persuasion. Cette vanité, assimilée à une vacuité, à un manque, équivaut à une capacité laissée à la grâce divine et destinée à être comblée. La lecture est offerte à l'âme comme un parcours dynamique, comme un cheminement spirituel. Le texte n'est en lui-même qu'une "vanité ", un miroir déformant qui ne renvoie pas toujours à une image fidèle à la réalité. La vanité n'est qu'une ombre, une fumée, qui passent et le lecteur, portant ses regards sur cet ensemble disparate, est convié à entrevoir les écarts, les fluctuations, les lignes fortes et dominantes.

L'œuvre, telle un miroir, reste ouverte sur le monde qu'elle dénonce et renvoie le lecteur à lui-même, à une éventuelle phase ascensionnelle pour accéder, à l'image de Jocelyne, à une forme de «limpidité, une fraîcheur d'âme " (Danaho, 1968, p. 43) qui l'autoriserait à reprendre à son tour ce discours aérien : 
J'aime beaucoup la montagne [...]. Elle n'encourage pas à l'amollissement, au farniente comme la mer. Mais elle est une véritable école d'énergie. Elle donne un sens aigu de la pureté. En effet, à chaque pas que l'on fait, à chaque effort que l'on accomplit pour gagner les hauteurs, on se sent comme plus léger. On a l'impression que l'on laisse derrière soi un peu de la pesanteur, de la lourdeur de la vie... (Danaho, 1968, p. 43-44)

\section{RÉFÉRENCES}

Bihan, A. (dir.). (2012). La Nouvelle-Calédonie, ses littératures et ses livres. Bibliographie sélective et commentée, $17^{\text {èmes }}$ Rencontres Francophones. Verson, France : Espace Senghor. Baudelaire, Ch. (2006). Les Fleurs du mal. Paris, France : Éditions Pocket.

Carco, F. (1933). L'ombre. Paris, France : Albin Michel.

Danaho, R. (1968). Chaque heure blesse. Paris, France : Éditions Promotion et Édition.

Genette, G. (1969). Figures II. Paris, France : Seuil.

Helms, L. (2018). Le personnage de roman. Paris, France : Armand Colin.

Hugo, V. (2002). Proses philosophiques, notices et notes d'Yves Gohin. Euvres complètes. Paris, France : Robert Laffont.

Jung, E. (2009). Francis Cargo, l'écrivain des minorités, des putains et des mauvais garçons. Carnets littéraires, chronique littéraire d'Éric Jung. Repéré de eric-yung.blogspot.com/.../ francis-carco-lecrivain-des-minorites.html

La Rochefoucauld. (1964). Réflexions ou sentences et maximes morales. Paris, France : Éditions Garnier-Flammarion.

Montaigne. (1965). Essais I, 3. Paris, France : Presses Universitaires de France.

Nietzsche, F. (1997). Euvres complètes. Paris, France : Robert Laffont.

Nietzsche, F. (2000). Le Gai Savoir. Dans Euvres. Paris, France : Flammarion.

Pascal, B. (1964). Pensées. Paris, France : Librairie philosophique J. Vrin.

Pétrarque. (1923), Canzoniere. Paris, France : Gallimard.

Rimbaud, A. (1873). Une saison en enfer. Bruxelles, Belgique : Alliance typographique (M.J. Poot et Compagnie).

Saint Augustin. (1864). Euvres complètes. Bar-le-Duc, France : L. Guérin \& Cie.

Valery, P. (1996). Tel quel. Paris, France : Gallimard.

RÉSUMÉ : La vanité, dans sa représentation la plus générale, nous invite à arrêter quelques instants notre course en avant, à mettre de côté nos divers agissements et à nous interroger sur notre destinée. La littérature, la peinture se focalisent souvent sur la fragilité de la vie. Elle est comparée à un souffle, à une vapeur qui paraît pour peu de temps. Les biens terrestres, les plaisirs mondains paraissent vains et éphémères et la quête incessante de l'homme toute aussi illusoire. Raoul Danaho, dans son roman Chaque heure blesse, publié en 1968, évoque l'histoire d'un jeune homme qui cherche sa propre identité et sa propre place dans le monde. Albert, cet homme originaire de Cayenne, s'est retrouvé à Paris, mais il n'y trouve pas la tranquillité souhaitée. 
La vie ne présente aucun attrait à ses yeux. Il doit « s'efforcer de vivre». Il se sent vide, incapable de fusionner avec le monde environnant et l'écriture de Danaho devient par là même fragmentaire comme pour " réfléchir » la vanité de l'existence. Albert cherchera-t-il " vainement » à " poursuivre sa route ", "ce tâtonnement dans le noir, cette marche aveugle dans la nuit » ou la rhétorique de la vanité se distendra-t-elle donc pour lui permettre de trouver une voie salutaire ? La Rochefoucauld énonçait avec une certaine acuité les propos suivants, renvoyant l'homme à lui-même, à ses semblables et au prisme réfléchissant : "Ce qui nous rend la vanité des autres insupportable, c'est qu'elle blesse la nôtre » $(1664,390)$. Nous sommes en droit de nous demander si la lumière parviendra à atteindre et à irradier le cœur humain.

Mots-clés : vanité, vie, mort, miroir, identité, écriture

\section{Vanity or mirror of the pretences in Every Hour Hurts by Raoul Danaho}

ABSTRACT: Vanity, in its most general representation, invites us to stop for a moment our race ahead, to set aside our various actions and to ask ourselves about our destiny. Literature, painting often focuses on the fragility of life. It is compared to a breath, a vapor that appears for a short time. Earthly goods, worldly pleasures seem vain and ephemeral and the incessant quest of man equally illusory. Raoul Danaho, in his novel Every Hour Hurts, published in 1968 evokes the story of a young man who seeks his own identity and his own place in the world. Albert, this man from Cayenne, ended up in Paris, but he did not find the desired tranquility. There is no attraction to life in his eyes. He must "strive to live". It feels empty, unable to merge with the surrounding world and the writing of Danaho becomes by the same fragmentary as to "reflect" the vanity of existence. Will Albert seek "vainly" to "continue his journey," "This groping in the dark, this blind march in the night," or is the rhetoric of vanity so distended to allow him to find a salutary way? The Rochefoucauld stated with some acuity the following words, referring the man to himself, to his fellows and to the reflecting prism: "What makes us the vanity of others unbearable is that it hurts ours" $(1664,390)$. We are entitled to ask ourselves whether the light will be able to reach and radiate the human heart.

Keywords: vanity, life, death, mirror, identity, writing 